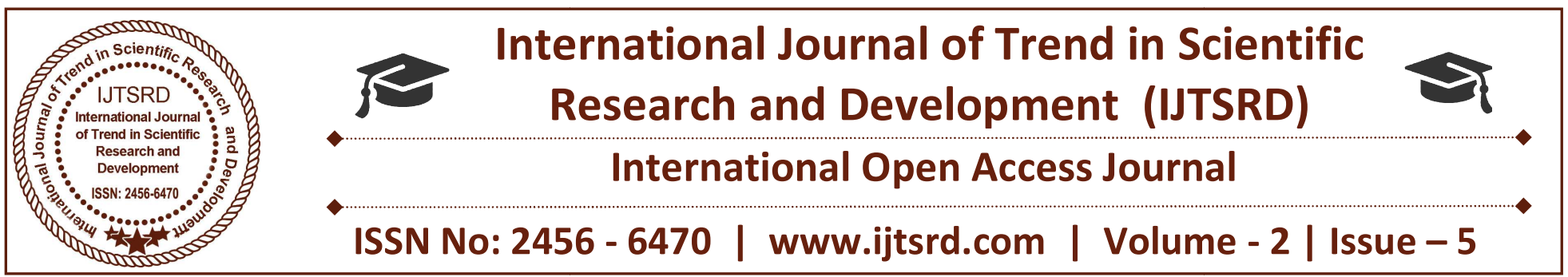

\title{
HPLC: Principle and Maintenance with Application
}

\author{
Yogesh Kumar, Sayed Md Mumtaz, Mustaq Ahmad \\ Research Scholar, Pharmacology Department, \\ Delhi Pharmaceutical Sciences and Research University Delhi, Delhi, India
}

\begin{abstract}
High performance liquid chromatography (HPLC) is a significant qualitative and quantitative technique, usually used for the estimation of pharmaceutical and biological samples. The chromatography term is derived from the Greek words namely chroma (colour) and graphein (to write). The chromatography is very accepted technique and it is mostly used analytically. It is the most resourceful, safest, reliable and fastest chromatographic technique for the quality control of drug components. This technique involves 2 phases' stationary and mobile phases. There are different types of chromatographic techniques. The separation of constituents is based on the variation between the partition coefficients of the two phases. This article is primed with an aim to review different aspects of HPLC, such as principle, types, instrumentation and application with maintenance.
\end{abstract}

KEYWORD: HPLC; Instrumentation; maintenance; application; chromatography

\section{INTRODUCTION}

Analytical chemistry is widely used in determining the qualitative and quantitative composition of typical mixtures. These two methods are the main aspects to understand the sample materials. Generally, analytical chemistry divides into two branches i.e. qualitative and quantitative analysis. High performance liquid chromatography also is known as High Pressure Liquid Chromatography. HPLC is an analysis technique used for separation, identification and quantification of typical mixtures such as organic, inorganic, biological, ionic and polymeric materials. HPLC is a type of column chromatography in which solvent flows with high pressure, so that sample can be separated into different constituents divided into different types based on modes of separation, the principle of separation, elution technique, scale of separation and based on the type of analysis [1]. The Detector is the heart of this instrument and the efficiency of this system depends upon detecting techniques. Many types of HPLC detectors such as UV-Vs, refractive index detector, photodiode detector, fluorescence, electrical conductivity

detector.

\section{A. Chromatography:}

Chromatography is an analytical method in which compounds are physically separated prior to measurement. The main purpose of chromatography is to separate and quantify the target sample in the matrix. The first developer of chromatography was $\mathrm{M}$. Tswett.

\section{B. Scope of HPLC:}

HPLC is used in various fields like in pharmaceuticals, biochemicals, food products, industrial chemicals, forensic chemistry, environmental field, clinical medicine for analysis for typical mixtures like antibiotics, amino acids, fatty acids, drugs, poisons, inorganic ions, urine extracts, oestrogens $[2,3]$.

\section{Need of HPLC}

The use of HPLC are increased day by day across the world due to its unique properties like high resolutions, high sensitivity (ppm-ppb), good repeatability, small sample size, moderate analysis condition, no need to vaporise the sample as in the gas chromatography, easy to fractionate the sample and purify $[4,5]$. 


\section{Instrumentation:}

The HPLC instrumentation involves pump, injector, column, detector, and integrator and display system.

Solvent reservoir: In HPLC the mobile phase or solvent is a mixture of polar and non-polar liquid components. Depending on the composition of the sample, the polar and non-polar solvents will be varied [6].

$>$ Pump: The pump suctions the mobile phase from the solvent reservoir and forces it to the column and then passes to the detector. $42000 \mathrm{KPa}$ is the operating pressure of the pump. This operating pressure depends on column dimensions, particle size, flow rate and composition of mobile phase [7].

> Sample Injector: An injector for an HPLC framework should give an infusion of the fluid specimen inside the scope of $0.1 \mathrm{~mL}$ to $100 \mathrm{~mL}$ of volume with high reproducibility and under high pressure (up to $4000 \mathrm{psi}$ ).

> Columns: Columns are typically made of cleaned stainless steel, are somewhere around $50 \mathrm{~mm}$ and $300 \mathrm{~mm}$ long and have an inward distance across of somewhere around 2 and $5 \mathrm{~mm}$. They are generally loaded with a stationary phase with a molecule size of $3 \mu \mathrm{m}$ to $10 \mu \mathrm{m}$ [8].

$>$ Detector: The HPLC detector, situated toward the end of the column distinguishes the analytes as they elute from the chromatographic column. Regularly utilized detectors are UV-spectroscopy, fluorescence, mass spectrometric SN and electrochemical identifiers [9].

> Data Collection Devices or Integrator: Signals from the detector might be gathered on graph recorders or electronic integrators that fluctuate in many-sided quality and in their capacity to process, store and reprocess chromatographic information. The PC coordinates the reaction of the indicator to every part and places it into a chromatograph that is anything but difficult to interpret [10].

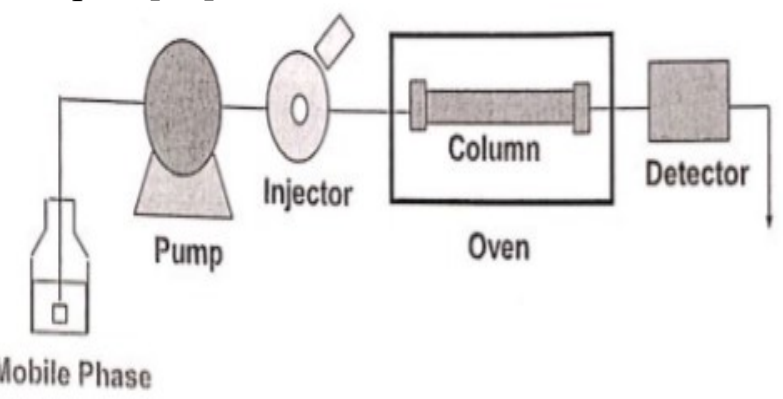

Fig.1. Flow diagram of HPLC
II. TYPES OF HPLC-

A. BASED ON MODE OF SEPARATION:

> Normal phase Chromatography: In this method, separation is based on polarity. The first developer used $\mathrm{CaCo}_{3}$ as separation column and Petroleum ether as developing solvent. The combination of stationary phase and mobile phase called normal phase chromatography. The stationary phase is polar, like silica gel type, cyanotype, amino type and mobile phase is non-polar example organic solvents, hexane, benzene [11].

> Reversed phase Chromatography: It is the reverse of the normal phase chromatography in which stationary phase is non- polar and mobile phase is polar. The example of the mobile phase are organic solvents (methanol, acetonitrile), buffer (phosphate buffer) [10, 12].

\section{B. Based on the principle of Separation: \\ $>$ Absorption Chromatography-}

In the absorption chromatography solute molecules bond directly to the surface of the stationary phase [13]. The component which has more affinity towards mobile phase elutes first and the component which has less affinity towards stationary phase elutes later. No two components have the same affinity towards mobile phase and stationary phase.

\section{$>$ Ion- exchange chromatography-}

Ion exchange chromatography is a process that allows the separation of ions and polar molecules based on their charge. It can be used for almost any kind of charged molecules including large proteins, small nucleotides and amino acids. Retention is based on the attraction between solute ions and charged sites bound to the stationary phase. Ions of the same charged are excluded. The use of resin (the stationary solid phase) is used to covalently attach anions or cations onto it. Solute ions of the opposite charge in the mobile liquid phase are attracted to the resin by electrostatic forces [10].

\section{Ion- Pair chromatography-}

It is a form of chromatography in which ions in solution can be "paired" or neutralized and separated as an ion pair on a reversed- phase column. Ionpairing agents are usually ionic compounds that contain a hydrocarbon chain that imparts a certain hydrophobicity so that the ion pair can be retained on a reversed-phase column [14].

\section{$>$ Gel permeation chromatography-}

This type of chromatography lacks an attractive interaction between the stationary phase and solute. 
The liquid or gaseous phase passes through a porous gel which separates the molecules according to its size. The pores are normally small and exclude the larger solute molecules, but allow smaller molecules to enter the gel, causing them to flow through a larger volume. This causes the larger molecules to pass through the column at a faster rate than the smaller ones [15].

\section{$>$ Affinity chromatography-}

This is the most selective type of chromatography employed. It utilizes the specific interaction between one kind of solute molecule and a second molecule that is immobilized on a stationary phase. For example, the immobilized molecule may be an antibody to some specific protein. When a solute containing a mixture of proteins are passed by this molecule, only the specific protein reacted to this antibody, binding it to the stationary phase. This protein is later extracted by changing the ionic strength or $\mathrm{pH}[16]$.

\section{Chiral chromatography-}

It involves the separation of stereoisomers. In the case of enantiomers, these have no chemical or physical differences apart from being three- dimensional mirror images. Conventional chromatography or other separation processes are incapable of separating them. To enable chiral separations to take place, either the mobile phase or the stationary phase must themselves be made chiral, giving differing affinities between the analytes [17].

\section{Based on elution technique- Isocratic elution:}

A separation in which the mobile phase composition remains constant throughout the procedure is termed isocratic elution. In isocratic elution, peak width increases with retention time linearly with the number of theoretical plates. This leads to the disadvantage that late-eluting peaks get very flat and broad. Best for simple separations. Often used in quality control application those supports and are in close proximity to a manufacturing process [18].

\section{$>$ Gradient elution-}

A separation in which the mobile phase composition is changed during the separation process is described as a gradient elution Gradient elution decreases the retention of the later-eluting components so that they elute faster, giving narrower peaks. This also improves the peak shape and the peak height. Best for the analysis of complex samples. Often used in method development for unknown mixtures. Linear gradients are most popular [19].

E. Based on a scale of operation-

$>$ Analytical HPLC: No recovery of individual components of a substance.

$>$ Preparative HPLC: Individual components of substance can be removed.

\section{F. Based on the type of Analysis-}

$>$ Qualitative analysis: Analysis of a substance in order to ascertain the nature of its chemical constituents and we can separate individual components but cannot assess the quantity in this analysis.

Quantitative analysis: Determining the amounts and proportions of its chemical constituents and the quantity of the impurity and individual components can be assessed.

Separation Mechanism: Compound are separated because the molecules are moves at different rates in the column.

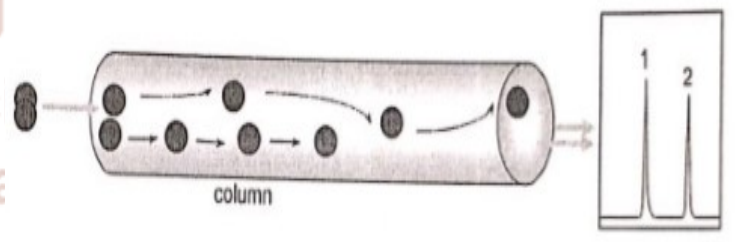

Fig.2. Separation mechanism

Due to different interactions between stationary phase and different sample, the molecules move to different rate, therefore separation can be done [20].

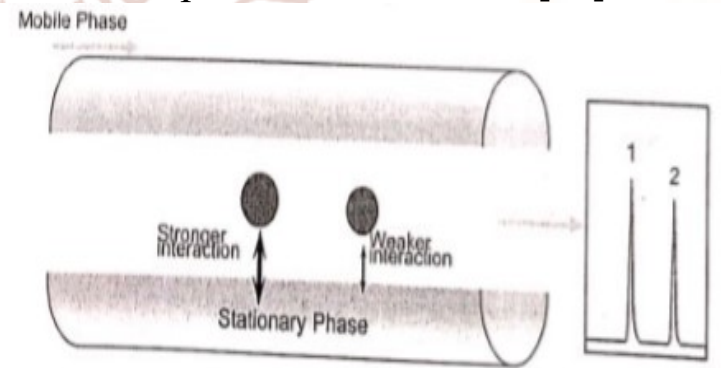

Fig.3. Separation mechanism

\section{DIFFERENT TYPES OF HPLC DETECTORS:}

Detectors in HPLC is placed at the end of the analytical column. The function of the detector is to examine the solution which is eluting from the column. An electronic signal is proportional to the concentration of individual components of the analyte. 
HPLC detector has particular characteristics such as excellent linear response as a function of the good sensitivity works approximately in the range of 0.01 $100 \mu \mathrm{g}$ of the compound in elutes.

A. UV-Visible- Ultraviolet Visible detector: It is the most commonly used detector in the HPLC. Most of the organic compounds absorb light in the region of UV (190-400nm) and in the visible region (400-750nm). It is based on Beer-Lambert law; deuterium and high-pressure xenon lamp are the sources of the UV. It has various advantages and disadvantage [21].

$>$ Advantages: It has high sensitivity, relative robust to temperature, compatible with gradient elution.

> Disadvantages: only compounds with UV or visible absorption could be detected.

B. Refractive index detectors: Refractive index is a bulk property of column eluent. In this detector, detection depends on solute modifying the overall refractive index of the mobile phase. Bulk property detectors have an inherently limited sensitivity. For the detection of the non-ionic compounds refractive index can be very useful and non-ionic compounds are not absorbed in the UV region and in fluoresce [22]. Various types of RI detectors are the following [23].

$>$ Christiansen effect detector

$>$ Interferometer detector

$>$ Thermal lens detector

$>$ Dielectric constant detector

$>$ Advantages:

1. Responds to nearly all solutes

2. Unaffected by the flow rate

\section{Disadvantages:}

1. Not as sensitive as most other types of detectors

2. Could not be used with a gradient elution

\section{Photodiode Array detector (PAD), Diode Array} detector: Photodiode arrays (semiconductor devices) are used in the detection unit. A DAD detects the absorption in UV to VIS region. While a UV-VIS detector has only one sample-side lightreceiving section, a DAD has multiple (1024 for L-2455/2455U) photodiode arrays to obtain information over a wide range of wavelengths at one time, which is a merit of the DAD [24].

\section{Advantages:}

1. .PDA Detector could analyze a sample simultaneously at many different wavelengths

2. UV visible spectra are useful for compound identification, checking peak purity, as well as finding the optimum absorbance for the compounds.

3. UV visible spectra of many compounds could be stored in the spectrum libraries, which are used for compound identification.

4. Relatively robust to temperature and flow rate fluctuations

5. Compatible with gradient elution.

\section{Disadvantages:}

1. Slightly less sensitive than the UV-Visible detector.

D. Fluorescence Detector: It is the most sensitive, specific detector among all existing HPLC detector. It is possible to detect the presence of single analyte molecule in the flow cell. The sensitivity of this detector is 10-1000 times higher than UV detector [25]. Various types of Fluorescence detector are:

The single wavelength excitation Fluorescence detector

Multi wavelength Fluorescence detector

Laser-induced Fluorescence detector

\section{Advantages:}

1. Sensitivity is higher than UV-Vis detector

2. Selectivity is high because of relatively fewcompounds fluorescences.

3. Compatible with gradient elution

\section{Disadvantages:}

1. Difficult to predict fluorescence

2. Greatly affected by environment, solvent, $\mathrm{pH}$, temperature, viscosity, ionic strength, dissolved gas.

E. Electrical Conductivity Detector: It provides universal, reproducible, high-sensitivity detection of all charged species such as anions, cations, metals, organic acids. These detectors measure the conductivity of the total mobile phase hence categorized in bulk density detectors. Electrodes of this detector are usually made up of platinum, stainless steel or some other noble metal [26]. 


\section{Advantages:}

1. Respond to ionic compounds and suitable for ion chromatography.

2. High sensitivity for low concentration range.

Disadvantages:

1. Sensitive to the fluctuations in the solvent flow and mobile phase composition

2. Not compatible with gradient elution.

\section{SELECTION OF DETECTOR:}

\section{Table1. Selection of Detector [27]}

\begin{tabular}{|c|c|c|}
\hline S.NO & DETECTORS & $\begin{array}{c}\text { Type of compounds can be } \\
\text { detected }\end{array}$ \\
\hline 1. & $\begin{array}{c}\text { UV-Vis } \\
\text { \&PDA }\end{array}$ & $\begin{array}{c}\text { Compounds with } \\
\text { chromophores, such as } \\
\text { aromatic rings or multiple } \\
\text { alternating double bonds. }\end{array}$ \\
\hline 2. & RF & $\begin{array}{c}\text { Fluorescent compounds, } \\
\text { usually with fused rings or } \\
\text { highly conjugated planar } \\
\text { system. }\end{array}$ \\
\hline 3. & CDD & $\begin{array}{c}\text { Charged compounds, such as } \\
\text { inorganic ions and organic } \\
\text { acids. }\end{array}$ \\
\hline 4. & ECD & $\begin{array}{c}\text { For easily oxidized IO } \\
\text { compounds like quinones or } \\
\text { amines. }\end{array}$ \\
\hline
\end{tabular}

\section{BASIC MAINTENANCE OF HPLC:}

A. Daily maintenance (pre-analysis check):

Filter mobile phase

$>$ Wait for mobile phase to reach room temperature

$>$ Purge all the flow lines with mobile phase

$>$ Replace mobile phase as necessary

$>$ If the solvent tubing is not completely filled with mobile phase, purge the flow line

$>$ After periods of disuse or changes in ambient temperature, purge the flow line.

$>$ If the buffer is used as mobile phase, wash the back of plunger seal.

$>$ If auto sampler is used, verify the auto sampler rinse the bottle fully.

$>$ Purge the autosampler rinse solution

$>$ Checks for leaks

$>$ Checks the pump pressure

$>$ Perform a baseline check.

\section{B. Daily maintenance (post-analysis checks):}

Wash the column

- If the column is not going to be used for a long time, take off it from the system after washing, and cap both ends of the column, store it in a cool and dark place.

$>$ Clean the flow line or the rest of the instrument, as necessary

Store the solvent reservoir filters in rinse solvent to prevent them from drying out

Solvent Reservoir Maintenance: The material of containers is glass which is generally used and plastic which has high sensitivity inorganic analysis.

1. Always cover the containers

2. Prevent the evaporation of solvents

3. Prevent the dust enter the mobile phase

4. Reduce the vapours in the room

5. Do not make containers as this may lead to the creation of partial vacuum and pump system.

6. Do not expose the bottle to direct sunlight/wind

7. Always keep reservoir above solvent delivery system, ensures good siphon feed to pump and prevents starving pumping system.

8. Always use a suction filter, prevent the particulates from reaching pumps and clean the suction filter by sonicating in isopropanol or $1 \mathrm{~N}$ nitric acid and water.

9. Good practice in the lab to label all bottles with composition inside as well as the date of prescription.

Mobile Phase: If the solvent has background absorption, the linear range will be smaller, the noise will be bigger and baseline stability, especially during gradient, runs will be poor.

1. The use of pure water systems is ideal as both inorganic and organic contaminants are removed.

2. Modern pure water systems give TOC values as indicated of total organic content in water.

3. Ghost peak will appear in gradient elution if water is bad quality.

\section{PRECAUTIONS IN HANDLING SOME HPLC GRADE ORGANIC SOLVENTS.}

1. Analytical grade THF contains butylated hydroxytoluene (BHT) as an oxidation inhibitor. As BHT has strong UV absorption, it is not added for HPLC grade THF.

2. As such, HPLC grade THF must be stored in cool and dark places to reduce oxidation to form peroxides. Accumulation of a large amount of organic peroxides may lead to explosions. 
A. Use of buffer solutions:

$>$ Buffer solutions must be filtered before use.

$>$ Buffer solutions must not be left in the system to avoid crystallization.

$>$ Effect on the pump- damage plunger and seal

$>$ Effect on the column- the creation of column voids

$>$ Effect on flow line- corrosion of stainless steel lines

$>$ Possible bacterial growth, especially phosphate buffers good medium for bacterial and fungus growth.

$>$ Ideally, solutions should be prepared fresh every day [28].

B. Premixing of Solvents: For isocratic systems, different solvents are premixed.

Example: to prepare a $1000 \mathrm{ml}$ of $50: 50$ mixture of water/methanol.

$>$ Measure out $500 \mathrm{ml}$ of water and $500 \mathrm{ml}$ of methanol separately, and then mix them together.

$>$ Measure $500 \mathrm{ml}$ of water and top up to the $1000 \mathrm{ml}$ mark of measuring cylinder/ volumetric flask with methanol.

$>$ Measure $500 \mathrm{ml}$ of methanol and top up to the $1000 \mathrm{ml}$ mark of measuring cylinder/ volumetric flask with water.

All three methods will give different compositions, whichever method selected must be used consistently for the repeatable result.

\section{Pump maintenance:}

Clean behind plunger seal when using buffer solution-

$>$ Automatic rinse kit for LC-10Avp and LC_20A series.

> Useful spares to be maintained- plunger seals, plunger, check valves, filter frit for line filter.

$>$ Most's tools required for maintenance work come with the pump accessories kit- do not lose them.

D. Sample treatment: Always filtered sample with $0.2 \mu \mathrm{m}$ or $0.45 \mu \mathrm{m}$ filters before injection. If the sample contains a lot of solid, centrifuge it, and the filter the solution.

$>$ Select suitable solvent to dissolve the sample. Normally dissolve sample in the mobile phase. But do not select a high soluble solvent as a sample solvent.

\section{E. Injector maintenance:}

Manual injector:

\section{Precautions:}

$>$ Do not use pointed or beveled needle tip. Use only flat and end type.

$>$ If $\mathrm{pH}$ higher than 10 is to be used, Delrin rotter seal is required.

When both manual and auto-injector are present in the system, auto sampler is always connected first, followed by manual.

$>$ Manual injector always set to load position when auto sampler is used and to inject position otherwise.

\section{F. Auto-Injector: General Maintenance}

Purge flow line at least once a day. Change purge liquid frequently.

Never used the salt solution as a purge liquid.

$>$ Used septums for sample vial recommended by the manufacturer only.

Septums should not be reused.

Do not fill vials to the brim.

G. Detector maintenance:

- UV-Vs detector: Check D2 lamp energy- Check the energy of reference cell. Change D2 lamp if the energy is lower than 800 .

(Note- do not touch the surface of D2 lamp by hand.)

- Waste bottle maintenance:

- Remember to empty waste bottle regularly.

- Do not mix concentrated nitric acid with an organic solvent.

- Do not immerse the drain tubing in the waste liquid as this may create back pressure on the flow cells [30].

\section{Advantages of HPLC-}

- High Resolution

- Good repeatability

- High Sensitivity (ppm-ppb)

- Small sample size

- Moderate analyses condition

- No need to vaporize the sample like GC

- Easy to fractionate the sample and purify

- Non-Destructive

- Separations fast and efficient (High Resolution Power)

- Continuous monitoring of the column effluent

- It can be applied to the separation and analysis of very complex mixtures.

- Accurate quantitative measurements 
- Repetitive and reproducible analysis using the same column

- Adsorption, partition, ion exchange and exclusion column separations are excellently made

- HPLC is more versatile than GLC in some respects because it has the advantage of not being restricted to volatile and thermally stable solute and the choice of mobile and stationary phases is much wider in HPLC.

- Both aqueous and non- aqueous samples can be analyzed with little or no sample pre-treatment

- A variety of solvents and column packing are available, providing a high degree of selectivity for specific analysis.

- It provides a means for determination of multiple components in a single analysis.

\section{Application-}

HPLC is one of the most widely applied analytical separation techniques-[29]

\section{Pharmaceutical:}

- Tablet dissolution of pharmaceutical dosages.

- Shelf life determination of pharmaceutical products.

- Identification of counterfeit drug products.

- Pharmaceutical quality control.

Environmental:

- Phenols in drinking water

- Identification of diphenhydramine in sediment samples.

- Bio monitoring of PAH pollution in high-altitude mountain lakes through the analysis of fish bile

- Estrogens in coastal waters- The sewage source

- Toxicity of tetracyclines and tetracycline degradation products to environmentally relevant bacteria

- Assessment of TNT toxicity in sediment.

\section{Forensics:}

- A mobile HPLC apparatus at dance parties- onsite identification and quantification of the drug Ecstasy.

- Identification of anabolic steroids in serum, urine, sweat and hair

- Forensic analysis of textile dyes

- Simultaneous quantification of psychotherapeutic drugs in human plasma

\section{$>$ Clinical:}

- Quantification of DEET in Human Urine.
- Analysis of antibiotics

- Increased urinary excretion of aquaporin 2 in patients with liver cirrhosis

- Detection of endogenous neuropeptides in brain extracellular fluids.

\section{$>$ Food \& Flavour:}

- Ensuring soft drink consistency and quality

- Analysis of vicinal dike tones in beer

- Sugar analysis in fruit juices

- Polycyclic aromatic hydrocarbons in Brazilian vegetables and fruits.

- Trace analysis of military high explosives in agricultural crops

- Stability of aspartame in the presence of glucose and vanillin $[2,10]$.

\section{CONCLUSION}

In this review, we include all fundamental aspects of HPLC technique. We describe the general technique of HPLC method and maintenance of the instrument. The general approach for these all point makes the precise quality of techniques. This method is widely accepted in the laboratory as well as in the clinical and forensic science lab due to the accuracy and precession and specificity with the HPLC. The only disadvantage of HPLC is high cost and complex methodology.

\section{REFERNCES:}

1. Olander, D.P., Instrumental Methods of Analysis, Sixth Edition (Willard, Hobart H.; Merritt, Lynne L.; Dean, John A.; Settle, Frank A., Jr.). Journal of Chemical Education, 1984. 61(8): p. A222.

2. Lipka, E. and C. Vaccher, Quantitative analysis of drugs in biological matrices by HPLC hyphenated to fluorescence detection. Bioanalysis, 2015. 7(6): p. 743-762.

3. Choudhury, H., et al., Development and validation of RP-HPLC method: scope of application in the determination of oil solubility of paclitaxel. J Chromatogr Sci, 2014. 52(1): p. 68-74.

4. Md Sabir, A., M. Moloy, and P. S Bhasin, HPLC METHOD DEVELOPMENT

AND

VALIDATION: A REVIEW. Vol. 4. 2015. 39-46.

5. Malherbe, C.J., D. de Beer, and E. Joubert, Development of on-line high performance liquid chromatography (HPLC)-biochemical detection methods as tools in the identification of bioactives. Int J Mol Sci, 2012. 13(3): p. 3101-33. 
6. Hopmann, E., W. Arlt, and M. Minceva, Solvent system selection in counter-current chromatography using conductor-like screening model for real solvents. J Chromatogr A, 2011. 1218(2): p. 242-50.

7. Xiang, Y., Y. Liu, and M.L. Lee, Ultrahigh pressure liquid chromatography using elevated temperature. J Chromatogr A, 2006. 1104(1-2): p. 198-202.

8. Martin, M. and G. Guiochon, Effects of high pressure in liquid chromatography. J Chromatogr A, 2005. 1090(1-2): p. 16-38.

9. Seger, C., S. Sturm, and H. Stuppner, Mass spectrometry and NMR spectroscopy: modern high-end detectors for high resolution separation techniques--state of the art in natural product HPLC-MS, HPLC-NMR, and CE-MS hyphenations. Nat Prod Rep, 2013. 30(7): p. 97087.

10. Malviya, R., et al., High performance liquid chromatography: A short review. Vol. 2. 2010. 22-26.

11. Lababidi, S. and W. Schrader, Online normalphase high-performance liquid chromatography/Fourier transform ion cyclotron resonance mass spectrometry: effects of different ionization methods on the characterization of highly complex crude oil mixtures. Rapid Commun Mass Spectrom, 2014. 28(12): p. 134552.

12. Fueangfung, S., Y. Yuan, and S. Fang, Denaturing reversed-phase HPLC using a mobile phase containing urea for oligodeoxynucleotide analysis. Nucleosides Nucleotides Nucleic Acids, 2014. 33(7): p. 481-8.

13. Shibasaki, H., et al., Separation and quantitative determination of 6alpha-hydroxycortisol and 6beta-hydroxycortisol in human urine by highperformance liquid chromatography with ultraviolet absorption detection. Anal Bioanal Chem, 2012. 402(9): p. 2945-52.

14. Jerz, G., et al., Separation of amaranthine-type betacyanins by ion-pair high-speed countercurrent chromatography. J Chromatogr A, 2014. 1344: p. 42-50.
15. O'Fagain, C., P.M. Cummins, and B.F. O'Connor, Gel-filtration chromatography. Methods Mol Biol, 2011. 681: p. 25-33.

16. Pfaunmiller, E.L., et al., Affinity monolith chromatography: a review of principles and recent analytical applications. Anal Bioanal Chem, 2013. 405(7): p. 2133-45.

17. Leon-Gonzalez, M.E., et al., Two-dimensional liquid chromatography for direct chiral separations: a review. Biomed Chromatogr, 2014. 28(1): p. 59-83.

18. Xu, M., et al., [Determination of chlorogenic acid and baicalin in Yinhuang film-coated tablets by HPLC isocratic elution]. Zhongguo Zhong Yao Za Zhi, 2013. 38(5): p. 691-3.

19. D'Archivio, A.A., M.A. Maggi, and F. Ruggieri, Prediction of the retention of s-triazines in reversed-phase high-performance liquid chromatography under linear gradient-elution conditions. J Sep Sci, 2014. 37(15): p. 1930-6.

20. Marchetti, N., et al., Determination of adsorption isotherms by means of HPLC: adsorption mechanism elucidation and separation optimization. J Sep Sci, 2009. 32(5-6): p. 727-41.

21. Kraiczek, K.G., et al., Highly flexible UV-vis radiation sources and novel detection schemes for spectrophotometric HPLC detection. Anal Chem, 2014. 86(2): p. 1146-52.

22. Ping, B.T.Y., H.A. Aziz, and Z. Idris, Comparison of Peak-area Ratios and Percentage Peak Area Derived from HPLC-evaporative Light Scattering and Refractive Index Detectors for Palm Oil and its Fractions. J Oleo Sci, 2018. 67(3): p. 265-272.

23. Kupina, S. and M. Roman, Determination of total carbohydrates in wine and wine-like beverages by HPLC with a refractive index detector: First Action 2013.12. J AOAC Int, 2014. 97(2): p. 498505 .

24. Pragst, F., M. Herzler, and B.T. Erxleben, Systematic toxicological analysis by highperformance liquid chromatography with diode array detection (HPLC-DAD). Clin Chem Lab Med, 2004. 42(11): p. 1325-40.

25. Raut, P.P. and S.Y. Charde, Simultaneous estimation of levodopa and carbidopa by RPHPLC using a fluorescence detector: its application to a pharmaceutical dosage form. Luminescence, 2014. 29(7): p. 762-71. 
26. Zhang, M., et al., Monitoring gradient profile online in micro- and nano-high performance liquid chromatography using conductivity detection. J Chromatogr A, 2016. 1460: p. 68-73.

27. Stubbs, C. and I. Kanfer, A stability-indicating high-performance liquid chromatographic assay of erythromycin estolate in pharmaceutical dosage forms. International Journal of Pharmaceutics, 1990. 63(2): p. 113-119.
28. Bakshi, M. and S. Singh, Development of validated stability-indicating assay methods-critical review. J Pharm Biomed Anal, 2002. 28(6): p. 1011-40.

29. Michalski, R., Application of ion chromatography in clinical studies and pharmaceutical industry. Mini Rev Med Chem, 2014. 14(10): p. 862-72.

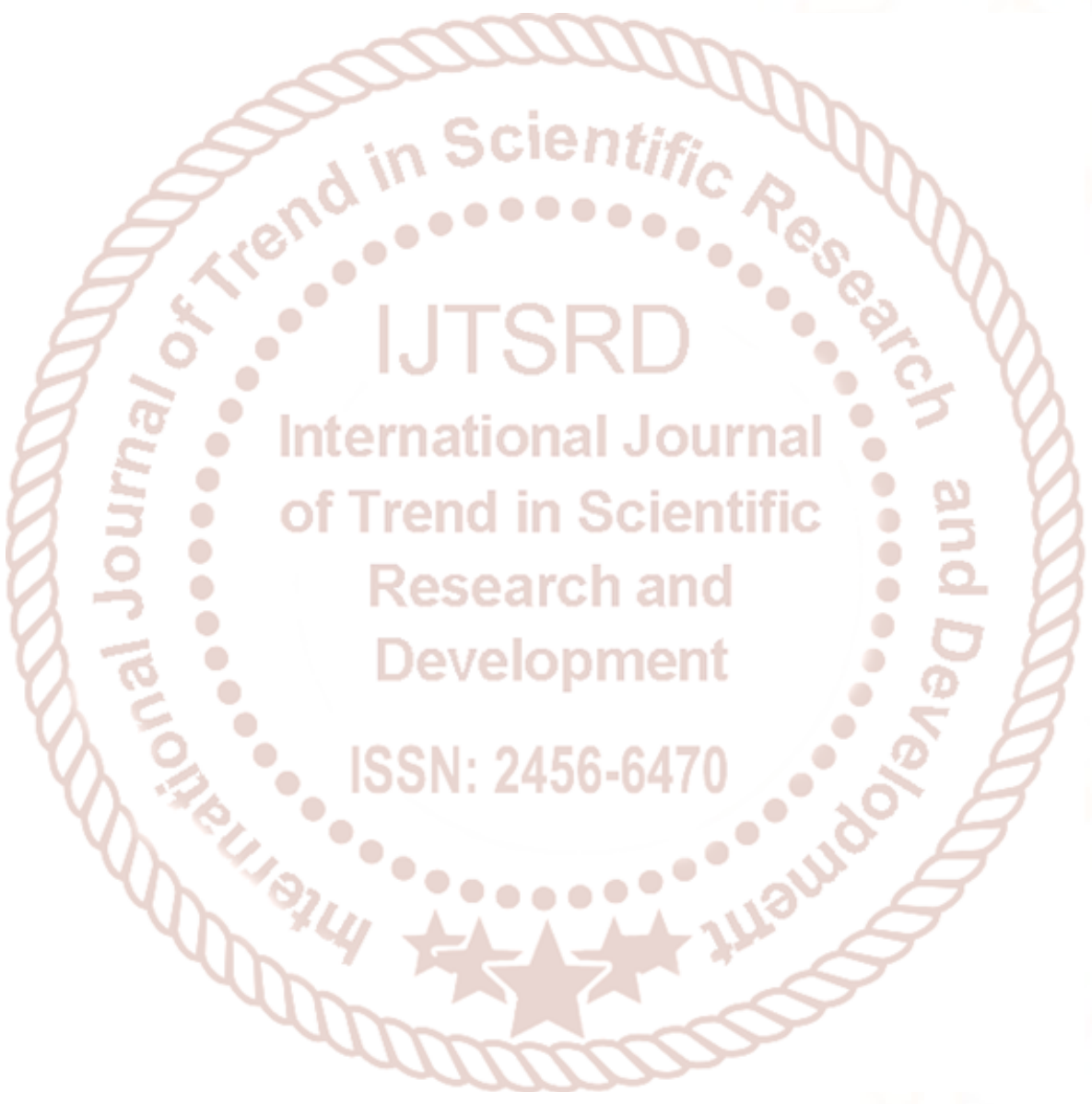

